\title{
Prosthetic Rehabilitation of a Huge Midfacial Defect Combined with Partial Mandibulectomy: A Clinical Report
}

\author{
H. Serdar Çötert and Hüseyin Kurtulmuş \\ Department of Prosthodontics, Faculty of Dentistry, Ege University, Bornova, İmir, Turkey
}

Correspondence should be addressed to: H. Serdar Çötert; cotert@gmail.com

Received date: 20 November 2013; Accepted date: 23 January 2014; Published date: 12 June 2015

Academic Editor: Daniela Micheline dos Santos

Copyright @ 2015 H. Serdar Çötert and Hüseyin Kurtulmuş. Distributed under Creative Commons CC-BY 4.0

\begin{abstract}
Carcinoma of the facial region often requires radical surgical excision, which results in varying amount of tissue loss of the facial structures. Such defects cause serious functional as well as esthetic complications. This paper describes the maxillofacial prosthetic rehabilitation of a patient having right lateral midfacial defect combined with a mandibular defect on the same side; by using a maxillary resection obturator, widened occlusal table and a facial prosthesis.
\end{abstract}

Keywords: Midfacial defect, mandibular defect, facial prosthesis, episthesis, polydimethyl siloxane, PDMS

\section{Introduction}

\section{Literature Review}

Advanced tumors of the maxillo-mandibular region occasionally require extensive surgical removal in order to eradicate the disease. The resulting surgical defect may involve the loss of both extraoral and intraoral structures, including portions of the nose, upper lip, cheek, and orbital contents. Also, segments of the maxilla, mandible, associated soft tissues, and teeth may be involved. Rodrigues et al. (2005) reported that, the functional impairment produced by such excisional procedures is severe. Brignoni and Dominici (2001) and NaBadalung (2003) also pointed out the disfigurement and functional impairment of the acquired mid-facial defects. Marunick et al. (1985) and Guttal et al. (2006) described the midfacial defects as to occur in the horizontal plane of the middle third of the face including two main categories: midline and lateral. Midline defects refer to the complete or partial involvement of the nose, and/or upper lip, along with an intraoral maxillary defects. A lateral defect may include complete or partial contents of the cheek and/or orbit, and may embrace an intraoral defect of the maxilla according to Hecker (2002). Pravinkumar and Patil (2010) reported that, midfacial defects usually require a facial prosthesis to restore function and appearance, and also, an intraoral prosthesis such as an obturator to restore speech and deglutition. Large facial defects involving the oral cavity can be difficult to restore prosthetically because of the lack of anatomical undercuts, limited means of retention, mobility of soft tissue margins, and the weight of the prosthesis. Functional disabilities in combination with the accompanying cosmetic disfigurement 
usually have a severe psychological impact on the patient. However, many patients with such surgical defects have been rehabilitated successfully with prosthetic restorations. The facial appearance of the patient, make participation in social activities conceivable.

Both the surgical and the maxillofacial prosthetic teams faced challenges during the rehabilitation of patients with tumor resective surgery involving the face, were described by Cheng et al. (1999) and Brignoni and Dominici (2001). NaBadalung (2003), Parr and Gardner (2003) and Rodrigues et al. (2005) reported that, the rehabilitation could be accomplished either surgically or prosthetically. According to NaBadalung (2003), the method of rehabilitation depends upon the site, size, etiology, severity, age, and expectations of the patient. In large midfacial defects, surgical reconstruction is almost impossible because of the size and extent of the deficiency. In addition to this, since a majority of the patients have extensive disease prior to surgery, radiation therapy may have been used as an adjunct. Therefore, in most of the patients, this fact precludes the possibility of surgical reconstruction. In the studies of NaBadalung (2003), Parr and Gardner (2003) and Rodrigues et al. (2005); general medical condition of the patient, anatomic complexity, possibility of recurrence, appearance of the area to be rehabilitated, complexity of the surgical procedure, and patient's refusal to undergo further surgery, were mentioned as the contraindications of the surgical reconstruction of a major defect. Cheng et al. (1999) reported that, the midfacial surgical defect might influence the patient's selfesteem as well as his or her body image. Brignoni and Dominici (2001) advocated that, large defects usually require a facial prosthesis to restore function and appearance. Because, prosthetic rehabilitation of such patients then has considerable advantages, in that a prosthesis offers the clinician and the patient the means to observe the healing wound for recurrence of the disease, esthetic improvement, technical simplicity, and inexpensive care.

Grossmann and Madjar (2004) reported that, an intraoral prosthesis such as an obturator is often needed to restore speech and swallowing. Thus, acquired maxillary defects can be restored to normal function and appearance. Separately, obturator prostheses continue to be the preferred method for the restoration of esthetics for most maxillectomy patients. Besides, obturator prosthesis should restore mastication, facial contours, and dental appearance according to Marunick (2004). Also Pigno and Funk (2001) reported that, the prosthetic prognosis of the management of maxillary defects primarily depends on the presence, condition and amount of remaining structures such as teeth, hard palate and facial tissues, functional status, motivation and adaptability of the patient, defect characteristics such as size, location, access, contour, available undercuts, and type of tissue, lining the defect. When these factors affect the prognosis, successful prosthetic management of the defect becomes more challenging according to the mentioned authors. For example, the restriction and the impaired flexibility of the reconstructed oral opening, make it difficult for the prosthodontist to fabricate accompanying intraoral prostheses, and make insertion and removal difficult for the patient. In addition, oral hygiene procedures will be compromised by the impaired oral access. However, oral function can be restored to impressive levels accompanied by an acceptable esthetic result, but there must be a solid foundation on which the oral prosthesis will be built. To accomplish this for partially edentulous patients, clinicians must provide comprehensive treatment planning and sound physiological design principles for a removable partial denture (RPD). According to Grossmann and Madjar (2004), framework designs of obturator prostheses should follow RPD design principles with modification for the unique requirements of the maxillectomy patients. Marunick (2004) advocated that, design principles must also consider the size and retentive qualities of the defect, access to the defect, and maximum oral opening along with clinical conditions dictating the definitive treatment plan, and RPD design which must be practical, affordable, and capable of meeting the functional needs and demands of the patient. 
Robinson and Rubright (1964), Desjardins (1979) and Sahin et al. (1993) are in agreement about the loss of continuity of the mandible destroying the balance of the lower face, and leading to decreased mandibular function by deviation of the residual segment toward the surgical site. Mandibular deviation and dysfunction were the major challenges of the prosthodontic treatment of the resected mandible according to Sahin et al. (1993). Curtis et al. (1975), Desjardins (1979), Moore and Mitchell (1976) and Sassen (1979) indicated that, the extent of mandibular resection and loss of continuity is directly related with the decreased masticatory function. Additionally, Schneider and Taylor (1986) and Mukohyama, (2004) pointed that, mandibulectomy patients suffer from numerous problems with speech and deglutition due to an anatomical compromise resulting from tumor resection. Conversely, patients with mandibular resections resulting in little soft tissue loss have less mandibular deviation according to Schneider and Taylor (1986) and Sahin et al. (1993). Articles of Robinson and Rubright (1964), Sassen (1979), Schneider and Taylor (1986), Aramany and Myers (1977) and Sahin et al. (1993) are in accordance about the surgical methods including maxillomandibular fixation and prosthodontics applications such as guidance restorations that might reduce mandibular deviation.

NaBadalung (2003) and Federspil (2009) reported that, several materials such as porcelain, natural rubber, gelatin, latex, polyurethanes and chlorinated polyethylene elastomers (CPE) have been employed in fabrication of the facial prostheses. But, two of them established themselves; methacrylates and polydimethyl siloxane (PDMS) elastomers. Methacrylates have the advantage of being more durable; however, they are relatively hard. NaBadalung (2003), Rodrigues et al. (2005), Montgomery and Kiat-Amnuay (2010) and Hatamleh et al. (2010) reported that, facial prostheses have been fabricated with PDMS elastomer since 50 years due to their lightweight and life-like appearance. Mostly, surgical retention provided by extraoral osseointegrated implants were preferred for facial prostheses according to Leonardi et al. (2008), Federspil (2009), Hatamleh et al. (2010) and Karakoca et al. (2010). However, the clinical study of Charpiot et al. (2006) showed that radiotherapy is a potential factor that reduces the rate of osseointegration. Ciocca et al. (2007) advised mechanical, Hatamleh et al. (2010) advised chemical and Shaikh (2011) and Padmanabhan (2012) advised anatomical retentive methods for such cases.

The present article reports the clinical and laboratory procedures for the maxillofacial prosthetic management of a case with a large midfacial defect complicated by hemimandibulectomy.

\section{Case Report}

A 43-year-old male has attended to the clinics of prosthetics. He was operated for right maxillary mesostructure resection due to an epidermoid carcinoma in 1993. Surgical, interim and definitive obturators were performed and followed periodically. He was operated again in 1994 for the maxillary superstructure resection including the exenteration of the right orbit due to the invasion of the neoplasm. Zygoma and cheek were also resected. The obturator prosthesis was renewed after the second surgery, and an eyeglass-frame retained facial prosthesis was made with a methacrylate material. In 2002, he underwent a third operation and, right ramus of the mandible including the condyle, coronoideus and angulus, were removed. Radiotherapy and chemotherapy were performed after surgery. Also, the obturator prosthesis was renewed. In 2012, recent prostheses were made.

Clinically, the orbit, orbital floor, zygoma, maxilla, and the mandible were absent on the right side. The extent of the defect was found as to present major esthetic and functional challenges when considering treatment with a facial prosthesis. Intraorally, there were only cuspid, and the second molar presents on the remaining left maxillary ridge. Mucosal quality over the remaining portion of the hard palate, and periodontal condition of the remaining teeth were examined clinically as well as radiographically, and were found to be healthy. Remaining maxillary structures provided minimal support, retention, and stability of a RPD maxillary obturator. Swallowing, chewing, 
deglutition and speech were damaged seriously. Mandibular condyle, collum, ramus, coronoideus, and corpus were also absent on the right side. The remaining mandibular structures were deviated medially.

The facial appearance of the patient was disfigured dramatically. Chewing, deglutition and speech intelligibility were affected drastically, as the patient's tongue could not effectively contact the palatal boundaries during deglutition and phonetics due to the lack of the anatomic continuity. As a result of the radiation therapy, long-term prognosis of the implant-retained prosthesis was poor as described by Charpiot et al. (2006). Hence, an anatomically and chemically retaining facial prosthesis from a PDMS elastomer, and a maxillary resection obturator with the widened occlusal table on the non-operated left side, were planned.

Maxillary resection obturator: Prior to the maxillary resection obturator, the remaining lower dentition was restored with metal ceramic fixed partial dentures (FPDs). Afterwards, preliminary impressions were made by using stock trays and irreversible hydrocolloid impression material (CA37, Cavex Dental BV, Haarlem, The Netherlands) and were poured in plaster to obtain diagnostic casts. Diagnostic upper cast was surveyed, and the path of insertion was determined taking the remaining teeth and the defect into consideration. To improve the retention efficacy of the clasps, metal ceramic veneers were made to the remaining cuspid and the second molar (Fig 1). Rest seats, reciprocal guide planes were milled according to the path of insertion of the obturator (Fig 2). A custom impression tray was fabricated and adjusted for proper extensions after the cementation of the crowns. The tray border was molded with green stick impression compound (Impression Compound, SpofaDental, Markova, Czech Republic). Final impression was made by polyvinyl-siloxane (Coltex Medium, Coltene-Whaledent, Altstatten, Switzerland). Final impression was boxed and poured in type III dental stone (DieKeen; Columbus Dental Stone, St. Louis, USA). A single-piece cast-metal framework was designed as described by Aramany (2001). Retaining and reciprocating clasps were cross-planned as described by Parr and Gardner (2003), Aramany (2001) and Firtell and Grisius (1980). The retaining clasps were designed as to place on the labial face of the cuspid and the palatinal face of the second molar. Opposing them, reciprocating clasps were placed (Fig 3).

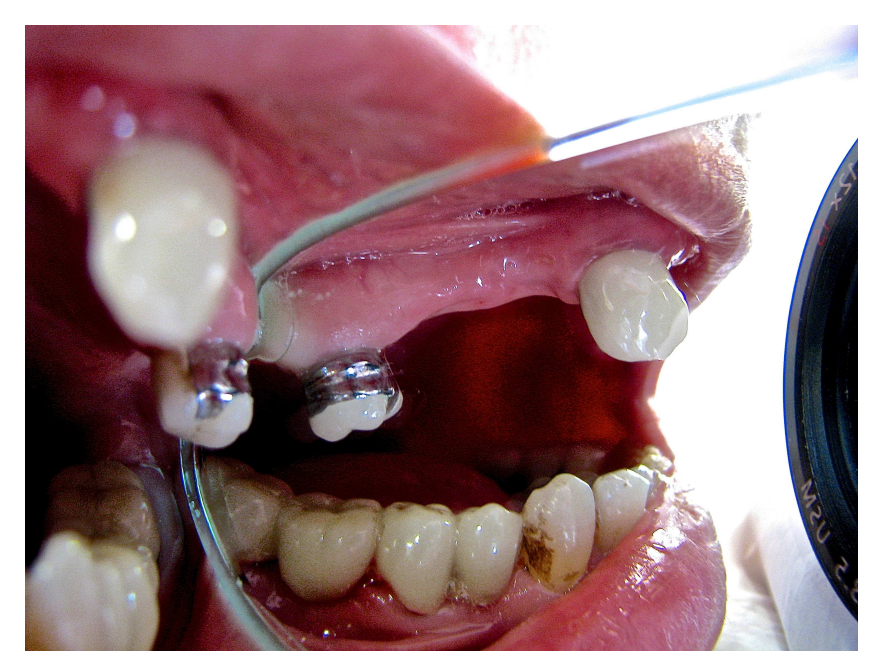

Fig 1. Buccal View of the Abutment Crowns. 


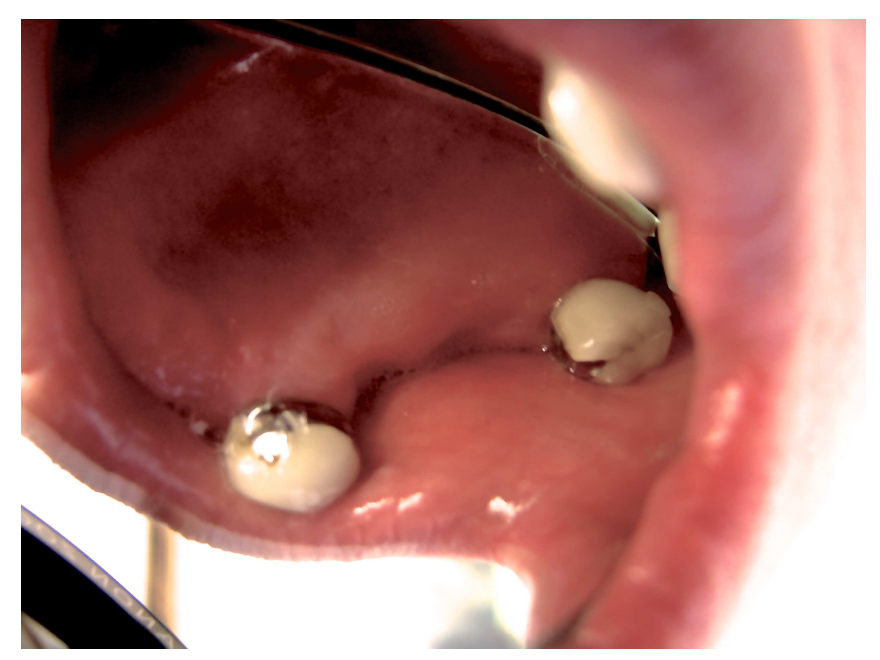

Fig 2. Palatal View of the Abutment Crowns.

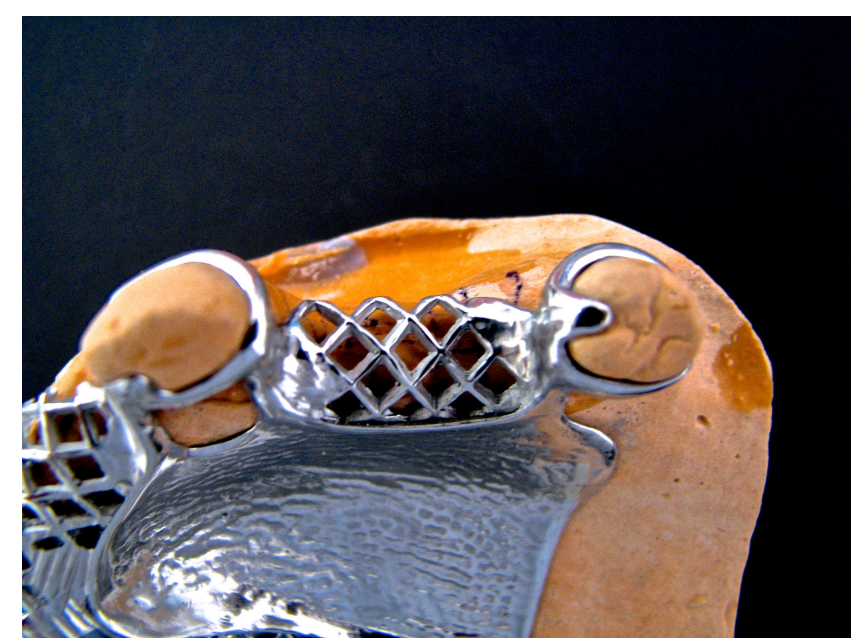

Fig 3. Cross-Planned Retaining and Reciprocating Elements of Cast-Metal Framework of the Obturator Prosthesis. Note the Retaining Clasp Arms on the Buccal Side of the Cuspid and the Palatal Side of the Molar, and the Reciprocating Arms on the Palatal Side of the Cuspid and the Buccal Side of the Molar.

The framework was cast in base metal alloy (Degussa Dental, Hanau, Germany). Maxillo mandibular relations were registered, artificial teeth were set, and the occlusal table of the non-operated left side was widened palatinally (Fig 4). An impression for the wide occlusal table was made with pink set-up wax (Pinnacle, Kerr, Mich, USA) with functionally generated path technique. A semi-adjustable articulator (Artex, Amann Girbach, Pforzheim, Germany) was employed for the mentioned procedures. The obturator was then processed with continuous pressure injection procedure as described by
Çötert et al. (2001), deflasked, trimmed and polished after polymerization. The obturator was then inserted into the mouth, adjusted, and oral hygiene instructions were given. The patient was pleased with the postoperative results of the treatment. Following placement of the maxillary obturator prosthesis, the patient noted that there were an accentuated compromise of the remaining lower lip support and postural position. The obturator prosthesis with a widened occlusal table was found to be comfortable and easy to maintain (Fig 5). 


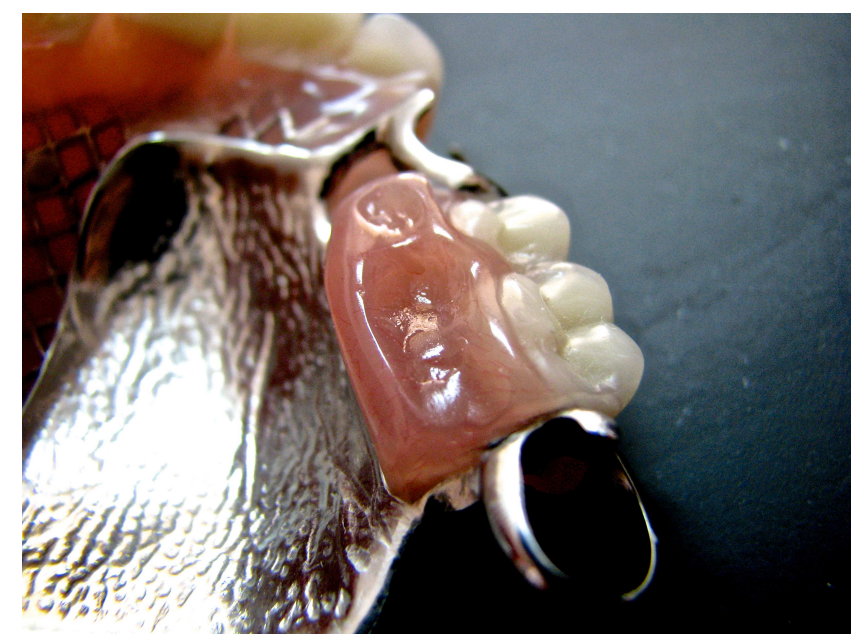

Fig 4. Widened Occlusal Table of the Obturator Prosthesis.

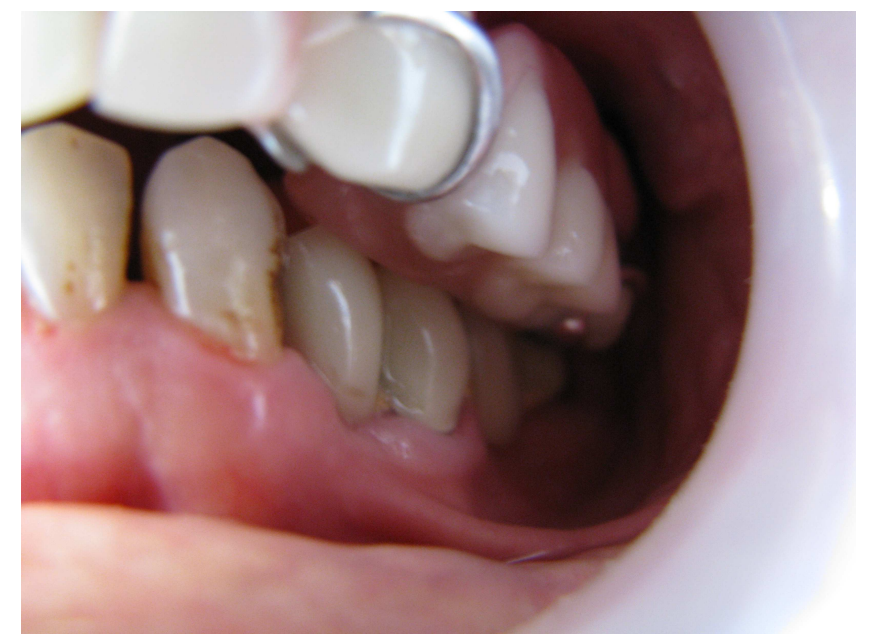

Fig 5. Widened Occlusal Table Occluding with the Posterior Dentition of the Medially Deviated Remaining Part of the Resected Mandible.

Facial prosthesis: An irreversible hydrocolloid facial-moulage was made to record the facial defect along with the surrounding normal extraoral structures, and the extraorally exposed portion of the obturator; and the moulage was cast with type III dental stone (Die-Keen; Columbus Dental Stone, St. Louis, MO) (Fig 6). 


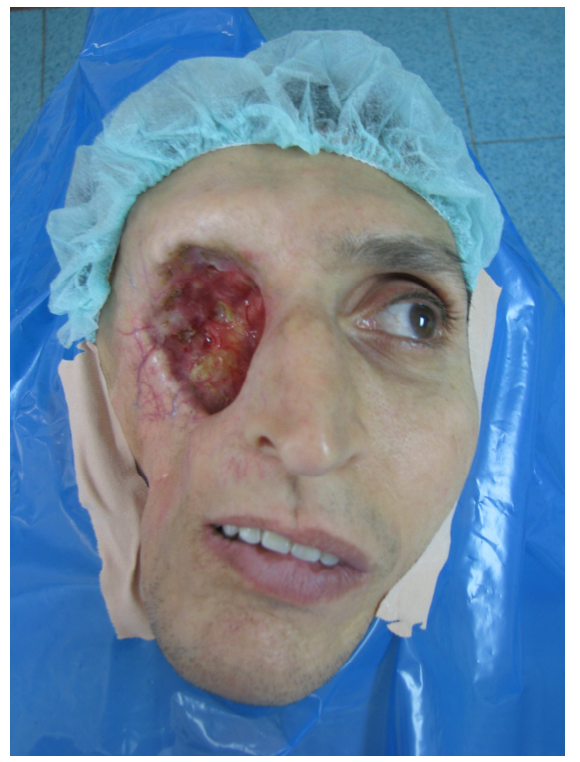

Fig 6. The Patient Exhibiting Midfacial Lateral Defect and Right Mandibular Defect, is Prepared for Facial Moulaging. Note the Defect, Facial Asymmetry and the Mandibular Deviation. Obturator Prosthesis in-situ.

The ocular bulb was fabricated in a conventional manner as described by Parr et al. (1983). The master cast was isolated (Separating Medium, Factor II Inc., Lakeside, AZ, USA), a wax prototype was carved from pink modeling wax (Lead Dent, Hamle Ltd. Şti., Izmir, Turkey). The individual ocular bulb was seated in wax prototype. A final tryin reconfirmed the ocular alignment in the defect. On completion, the wax prototype was verified at the trial insertion appointment. The prototype was then flasked, the wax was eliminated and the plaster surfaces were isolated (Separating Medium, Factor II Inc., Lakeside, AZ, USA). PDMS material (VST 50, Factor II Inc., Lakeside, AZ, USA) was colored with intrinsic stains (KT-699, Silicone Coloring Kit; Factor II Inc, Lakeside, AZ, USA) according to the patient's skin color, prepared, packed and polymerized according to the recommendations of the manufacturer. Then, the deflasked prosthesis was trimmed, cleaned and tried in-situ. It was finally colorfinished with oil pigments (Factor II Inc, Lakeside, AZ, USA) externally.

To improve the retention further, a medical adhesive (Secure Medical Adhesive; Factor II Inc, Lakeside, AZ, USA) was used on the face, and the prosthesis was inserted into the defect (Fig 7). The patient was instructed on home care and prosthesis maintenance, and attended recall visits every 6 months. During these visits, the obturator and facial prosthesis were thoroughly cleaned and disinfected (MD 520; Dürr Dental GmbH, Bietigheim-Bissingen, Germany). 


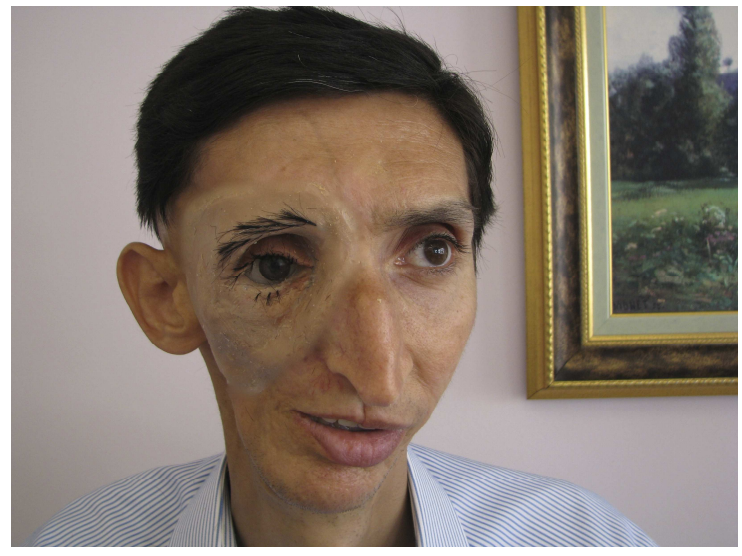

Fig 7. Polydimethyl Siloxane Elastomeric Facial Prosthesis in-situ.

Prosthesis İs still in service and the patient İS pleased after twelve months of insertion. Improvements in esthetics, speak intelligibility, chewing and deglutition are achieved.

\section{Discussion}

Prosthetic treatment of a patient having a huge maxillofacial defect was described in the present paper. The patient was exhibiting a midfacial lateral defect including the absence of the maxillary and orbital tissues. Moreover, the functions and the abilities were more complicated due to the mediotrusion of the remaining mandible due to the mandibular resection. Such large maxillofacial defects have not been reported frequently in maxillofacial prosthetic literature. Federspil (2009) reported that, the large maxillo-facial defects could result in serious functional impairment of speech, mastication, and swallowing. The cosmetic disfigurement often has a significant psychological impact. Acceptable esthetic appearance usually can be regained. With ingenuity and an understanding of the remaining anatomic structures, intraoral and extraoral prostheses that mutually retain one another, can be constructed. Retention of facial prostheses can be maintained in four ways according to the mentioned author: anatomic, mechanic, chemical and surgical retentions. Surgical retention provided by extraoral osseointegrated implants were reported as more suitable for facial prostheses by Leonardi et al. (2008), Federspil (2009), Hatamleh et al. (2010) and Karakoca et al. (2010). However, radiotherapy is known to be a potential factor that reduces the rate of osseointegration as demonstrated by Charpiot et al. (2006). Ciocca et al. (2007) advised mechanical retention in some circumstances. Chemical retention was advised by Hatamleh et al. (2010). Also, anatomical retention was advised by Shaikh (2011) and Padmanabhan (2012). Hatamleh et al. (2010) reported that the ocular prostheses included in their study were entirely retained by postoperative anatomical undercuts. They also reported that the adhesives are commonly used for orbital prostheses (48\%). Contrarily, Hecker (2002) has not advised skin adhesives for large defects, especially for intraoral integrated midfacials, due to the presence of persistent moisture and saliva. Additionally, weight reduction resulting from the use of hollow prosthesis was found to be profitable, and might contribute to the retention by Hecker (2002) and Pravinkumar and Patil (2010).

Chemical and anatomical retentive methods were preferred for the recent case, as advised in the reports of Charpiot et al. (2006), Ciocca et al. (2007), Hatamleh et al. (2010), Shaikh (2011) and Padmanabhan (2012). Reliable support and retention were obtained from the prominent undercuts of the postoperative defect cavity. Skin adhesive was employed for camouflaging the marginal irregularities during the facial gestures. Width of the upper lip and its musculature were observed as protecting the bonded margins from the oral liquids. Weight reductive approaches such as hollow 
design, were found unnecessary due to the prominent undercuts.

Aziz et al. (2003) and Kurunmäki et al. (2008) reported that, a number of biomaterials and techniques have been used in the fabrication of facial prostheses for several decades; and each material has its advantages and shortcomings. For the purpose of prosthetic rehabilitation for facial defects, biomaterials such as methacrylates, PET, polyurethane, and PDMS elastomers have been utilized. PDMS elastomers are generally used for facial prostheses because of their various superior features according to Montgomery and Kiat-Amnuay (2010) and Hatamleh et al. (2010). The most important advantages of PDMS elastomers were reported as good esthetics ease of coloring, easy manipulation, possibility of thin margins, and adhesive compatibility as described by Aziz et al. (2003), Kurunmäki et al. (2008). On the other hand, discoloration over time, technique-sensitivity, and lack of reparability, extrinsic colors peel/fade, and lack of longevity may be listed as the disadvantages. Instability and rapid degradation were mentioned as the limitations of the material by Aziz et al. (2003) and Kurunmäki et al. (2008).

Grossmann and Madjar (2004) advocated that, the biomechanical principles remain unchanged for maxillary obturator retention. One option to achieve the requirements is to prepare the abutments to receive complete coverage. Consequently, abutment contours may be modified. In this study, metal ceramic veneers having cross-planned reciprocal clasp guides and retentive clasp seats, were employed to improve the retention and stability of the obturator prosthesis. Clasp assemblies of the obturator infrastructure were properly engaged to the abutments. Cross-placement of the retaining and the reciprocating arms of the clasp systems was described in previous papers of Firtell and Grisius (1980), Aramany (2001) and Parr and Gardner (2003).

Deviation of the remaining mandible after surgical resection, was described by Robinson and Rubright (1964), Desjardins (1979) and Sahin et al. (1993). According to Curtis et al. (1975), Moore and Mitchell
(1976), Desjardins (1979) and Sahin et al. (1993); this deviation destroys both centric and eccentric relations and decreases masticatory performance. Schneider and Taylor (1986) and Rodrigues et al. (2005) reported that, mandibular resection procedure also affects the oral functions such as speech and deglutition. Various prosthodontic appliances guiding the remaining mandible to a functionally acceptable centric relation were described in reviewed papers of Curtis et al. (1975), Schneider and Taylor (1986) and Sahin et al. (1993). Sahin et al. (1993) advocated that, the success of mandibular guidance therapy depends upon the nature of the surgical defect, early initiation of guidance therapy, patient cooperation, and other factors. According to Aramany and Myers (1977), mandible guidance therapy was found to be most successful in patients' resection involved only bony structures, with minimal sacrifice of tongue, floor of the mouth, and adjacent soft tissues. In the present case, guidance treatment was not preferred due to the extent of the resection, severely affected regional musculature, postoperative scars and tissue differentiations following radiotherapy. For this reason, occlusal table of the upper left dental arch was widened palatinally to obtain an occlusal surface, so that the dentition of the remaining mandible may occlude.

After 12 months the resection obturator and the widened occlusal table were still functional; chewing and swallowing performances were acceptable, preservation of the body weight was good, speaking intelligibility was good.

\section{Summary}

Carcinoma of the facial region often requires radical surgical excision, which results in varying amount of tissue loss of the facial structures. Such defects cause serious functional as well as esthetic complications. This paper describes the prosthetic rehabilitation of a patient having right lateral midfacial defect combined with a mandibular defect on the same side. Treatment consists a facial prosthesis and a maxillary resection obturator has a widened occlusal table occluding with remaining mandibular teeth 


\section{References}

1. Rodrigues, S., Shenoy, V. K. \& Shenoy, K. (2005). "Prosthetic Rehabilitation of a Patient after Partial Rhinectomy: A Clinical Report," The Journal of Prosthetic Dentistry 93 (2): 125-8.

2. NaBadalung, D. P. (2003). "Prosthetic Rehabilitation of a Total Rhinectomy Patient Resulting from Squamous Cell Carcinoma of the Nasal Septum: A Clinical Report," The Journal of Prosthetic Dentistry 89 (3): 234-8.

3. Brignoni, R. \& Dominici, J. T. (2001). “An Intraoral-Extraoral Combination Prosthesis Using an Intermediate Framework and Magnets: A Clinical Report," The Journal of Prosthetic Dentistry 85 (1) :7-11.

4. Marunick, M. T., Harrison, R. \& Beumer, J. (1985). "Prosthetic Rehabilitation of Midfacial Defects," The Journal of Prosthetic Dentistry 54 (4): 533-8.

5. Guttal, S. S., Patil, N. P. \& Shetye, A. D. (2006). "Prosthetic Rehabilitation of a Midfacial Defect Resulting from Lethal Midline Granuloma-A Clinical Report," Journal of Oral Rehabilitation 33 (11): 863-7.

6. Hecker, D. M. (2002). "Maxillofacial Rehabilitation of a Large Facial Defect Resulting from an Arteriovenous Malformation Utilizing a Two-Piece Prosthesis," The Journal of Prosthetic Dentistry 89 (2): 109-13.

7. Pravinkumar, G. \& Patil, M. D. S. (2010). "Modified Technique to Fabricate a Hollow Light-Weight Facial Prosthesis for Lateral Midfacial Defect: A Clinical Report," The Journal of Advanced Prosthodontics 2 (3): 6570.

8. Cheng, A. C., Morrison, D., Wee, A. G., Maxymiw, W. G. \& Archibald, D. (1999). "Maxillofacial Prosthodontic Management of a Facial Defect Complicated by a Necrotic Frontal Bone Flap: A Clinical Report," The Journal of Prosthetic Dentistry 82 (1): 3-7.

9. Parr, G. R. \& Gardner, L. K. (2003). "The Evaluation of the Obturator Framework
Design," The Journal of Prosthetic Dentistry 89 (6): 608-10.

10. Grossmann, Y. \& Madjar, D. (2004). "Resin-Bonded Attachments for Maxillary Obturator Retention: A Clinical Report," The Journal of Prosthetic Dentistry 92 (6): 229-32.

11. Marunick, M. (2004). "Hybrid Gate Design Framework for the Rehabilitation of the Maxillectomy Patient," The Journal of Prosthetic Dentistry 91 (4): 315-8.

12. Pigno, M. A. \& Funk, J. J. (2001). "Augmentation of Obturator Retention by Extension into the Nasal Aperture: A Clinical Report," The Journal of Prosthetic Dentistry 85 (4): 349-51.

13. Sahin, N., Hekimoglu, C. \& Aslan, Y. (1993). "The Fabrication of Cast Metal Guidance Flange Prostheses for a Patient with Segmental Mandibulectomy: A Clinical Report," The Journal of Prosthetic Dentistry 93 (3): 217-20.

14. Desjardins, R. P. (1979). "Occlusal Consideration for the Partial Mandibulectomy Patient," The Journal of Prosthetic Dentistry 41 (3): 308-15.

15. Robinson, J. E. \& Rubright, W. C. (1964). "Use of a Guide Plane for Maintaining the Residual Fragment in Partial or Hemimandibulectomy," The Journal of Prosthetic Dentistry 14: 992-9.

16. Curtis, T. A., Taylor, R. C. \& Rositano, S. A. (1975). "Physical Problems in Obtaining Records of the Maxillofacial Patients," The Journal of Prosthetic Dentistry 34 (5): 539-43.

17. Sassen, H. (1979). "Prosthetic Treatment Following Partial Resection of the MandibleA Case Report," Quintessence International 1:31-36.

18. Moore, D. J. \& Mitchell, D. L. (1976). "Rehabilitating Dentulous Hemimandibulectomy Patients," The Journal of Prosthetic Dentistry 35 (2): 202-6.

19. Mukohyama, H., Kadota, C., Ohyama, T. \& Taniguchi, H. (2004). "Lip Plumper Prosthesis for a Patient with a Marginal 
Mandibulectomy: A Clinical Report," The Journal of Prosthetic Dentistry 92 (1): 23-6.

20. Schneider, R. L. \& Taylor, T. D. (1986). "Mandibular Resection Guidance Prostheses. A Literature Review," The Journal of Prosthetic Dentistry 55 (1): 84-6.

21. Aramany, M. A. \& Myers, E. N. (1977). "Intermaxillary Fixation Following Mandibular Resection," The Journal of Prosthetic Dentistry 37 (4): 437-44.

22. Federspil, P. A. (2009). "Implant-Retained Craniofacial Prostheses for Facial Defects," Laringorhinootologie 88 (Suppl 1): 125-38.

23. Montgomery, P. C. \& Kiat-Amnuay, S. (2010). "Survey of Currently Used Materials for Fabrication of Extraoral Maxillofacial Prostheses in North America, Europe, Asia, and Australia," Journal of Prosthodontics 19 (6): 482-90.

24. Hatamleh, M. M., Haylock, C., Watson, J. \& Watts, D. C. (2010). "Maxillofacial Prosthetic Rehabilitation in the UK: A Survey of Maxillofacial Prosthetists' and Technologists' Attitudes and Opinions," International Journal of Oral \& Maxillofacial Surgery 39 (12):1186-92.

25. Leonardi, A., Buonaccorsi, S., Pellacchia, V., Moricca, L. M., Indrizzi, E. \& Fini, G. (2008). "Maxillofacial Prosthetic Rehabilitation Using Extraoral Implants," Journal of Craniofacial Surgery 19 (2): 398405.

26. Karakoca, S., Aydin, C., Yilmaz, H. \& Bal, B. T. (2010). "Retrospective Study of Treatment Outcomes with Implant-Retained Extraoral Prostheses: Survival Rates and Prosthetic Complications," The Journal of Prosthetic Dentistry 103 (2): 118-26.

27. Charpiot, A., Chambres, O., Herve, J. F., Million, P., Riedinger, A. M. \& Hemar, P. (2006). "Osteointegrated Cranio-Facial Implants: 49 Patients Report," Revue de Laryngologie- Otologie- Rhinologie 127 (4): 217-22.
28. Ciocca, L., Maremonti, P., Bianchi, B. \& Scotti, R. (2007). “Maxillofacial Rehabilitation after Rhinectomy Using Two Different Treatment Options: Clinical Reports," Journal of Oral Rehabilitation 34 (4): 311-5.

29. Padmanabhan, T. V., Mohamed, K., Parameswari, D. \& Nitin, S. K. (2012). "Prosthetic Rehabilitation of an Orbital and Facial Defect: A Clinical Report," Journal of Prosthodontics 21 (3): 200-4.

30. Shaikh, S. R., Patil, P. G. \& Puri, S. (2011). "A Modified Technique for Retention of Orbital Prosthesis," Indian Journal of Dental Research 22 (6): 863-5.

31. Aramany, M. A. (2001). "Basic Principles of Obturator Design for Partially Edentulous Patients. Part I. Classification," (Classical Article) The Journal of Prosthetic Dentistry 86 (6): 559-61.

32. Firtell, D. N. \& Grisius, R. J. (1980). "Retention of Obturator-Removable Partial Dentures: A Comparison of Buccal and Lingual Retention," The Journal of Prosthetic Dentistry 43 (2): 212-7.

33. Çötert, H. S., Cura, C. \& Kesercioğlu, A. (2001). "Modified Flasking Technique for Processing a Maxillary Resection Obturator with Continuous Pressure Injection," The Journal of Prosthetic Dentistry 86 (4): 438-40.

34. Parr, G. R., Goldmann, B. M. \& Rahn, A. O. (1983). "Prosthetic Treatment of Ocular and Orbital Defects," The Journal of Prosthetic Dentistry 49 (3): 379-83.

35. Aziz, T., Waters, M. \& Jagger, R. (2003). "Analysis of the Properties of Silicone Rubber Maxillofacial Prosthetic Materials," Journal of Dentistry 31 (1): 67-74.

36. Kurunmäki, H., Kantola, R., Hatamleh, M. M., Watts, D. C. \& Vallittu, P. K. (2008). "A Fiber-Reinforced Composite Prosthesis Restoring a Lateral Midfacial Defect: A Clinical Report," The Journal of Prosthetic Dentistry 100 (5): 348-52. 